\title{
SAFETY OF HONEY CONSUMED IN ENUGU STATE, NIGERIA: A PUBLIC HEALTH RISK ASSESSMENT OF LEAD AND POLYCYCLIC AROMATIC HYDROCARBONS
}

\author{
Harrison Anezi Ozoani ${ }^{1}$, Anthonet Ndidiamaka Ezejiofor ${ }^{1,2}$, Cecilia Nwadiuto Amadi ${ }^{1}$ \\ Ifeyinwa Chijioke-Nwauche $e^{3}$ and Orish Ebere Orisakwe ${ }^{1,2}$
}

\author{
${ }^{1}$ Department of Experimental Pharmacology \& Toxicology, Faculty of Pharmacy, \\ University of Port Harcourt, PMB, 5323 Port Harcourt, Rivers State, Nigeria, \\ ${ }^{2}$ World Bank Africa, Centre of Excellence in Public Health and Toxicological Research (PUTOR), \\ University of Port Harcourt, PMB,5323 Port Harcourt, Rivers State, Nigeria \\ ${ }^{3}$ Department of Clinical Pharmacy, Faculty of Pharmacy, University of Port Harcourt, \\ PMB, 5323 Port Harcourt, Rivers State, Nigeria
}

\begin{abstract}
Background: Information about lead $(\mathrm{Pb})$ and polycyclic aromatic hydrocarbons (PAHs) concentrations in honey and their dietary intake is very important in human health risk assessment. Currently, there are paucity of data on the risk assessment and concentrations of lead and PAHs in honey in Nigeria.

Objective: This study has determined the potential human health risk of lead and PAHs associated with the consumption of honey in Enugu state, Nigeria.

Materials and methods: Lead and US EPA 16 priority PAHs in honey harvested from rural and urban communities in March 2016 were determined using Atomic Absorption Spectrometer and Gas Chromatograph respectively. Carcinogenic and non-carcinogenic risk assessments were carried.

Results: The mean concentration of $\mathrm{Pb}$ in honey ranged from $0.005 \mathrm{mg} / \mathrm{kg}-0.08 \mathrm{mg} / \mathrm{kg}$. The mean concentrations of 16 PAHs in honey ranged from $4.71 \mathrm{E}-03-2.72 \mathrm{mg} / \mathrm{kg}$. The dietary intake of the PAHs for adults and children ranged from $0.0021-0.0259 \mathrm{mg} / \mathrm{kg} /$ day and $0.0011-0.0129 \mathrm{mg} / \mathrm{kg} /$ day respectively. The levels of BaPeq and their margin of exposure MOE suggest non-significant health risk. The incremental life cancer risk ILCR of Pb were within safe range of $<1 \mathrm{E}-04$.

Conclusion: Consumption of honey from Enugu State, Nigeria may not pose a significant health risk.
\end{abstract}

Key words: risk assessment, lead, polycyclic aromatic hydrocarbons, public health

\section{INTRODUCTION}

The occurrence of polycyclic aromatic hydrocarbons (PAHs), a group of chemicals with two or more aromatic rings, in the environment and in food is of concern as some have been proven to be carcinogenic [1,2]. Usually foods represent the major source of exposure. In 2002, the Scientific Committee on Food (SCF) recommended the monitoring of 15 PAHs with a conclusion that 15 out of 33 investigated PAHs, show mutagenic/genotoxic effects and, with exception of benzo[ghi]perylene, have also shown clear carcinogenic effects [2]. The need for the continuous data collection on other PAHs levels in foods for risk assessments has been stressed by SCF.
Lead $(\mathrm{Pb})$, a multi organ toxicant and one of the most ubiquitous environmental contaminants which can be harmful even in low concentrations due to its ability to accumulate in different parts of human body [3, 4, 5]. Lead is non-biodegradable and persists in nature [5]. Ingestion of lead can seriously cause depletion of some essential ultra-trace elements like selenium and zinc attendant immunological consequences, intrauterine growth retardation, psychosocial dysfunctions, disabilities association with malnutrition and cancer [6]

Honey is a sweet viscous liquid produced by bees that serves both as food and medicine [7, 8]. It contains carbohydrate, proteins, minerals, trace elements, vitamins, aroma compounds and polyphenols. The composition of

Corresponding author: Orish Ebere Orisakwe, Department of Experimental Pharmacology \& Toxicology, Faculty of Pharmacy, University of Port-Harcourt, Rivers State, Nigeria, e-mail: orishebere@gmail.com 
honey depends greatly on the botanical origin; climate, environment, soil, manner of harvest, and storage conditions [9]. Bees travel over long distances during forage and are exposed frequently through air, soil, water, vegetation etc, to accumulate various contaminants seen in the honey they produce. Consequently, metal levels and other contaminants in honey have been recognized as biological indicators of environmental pollution [10].

Lead and polycyclic aromatic hydrocarbons may coexist in diverse environments including foods and have been implicated in mutagenesis, carcinogenesis, and teratogenesis [11].

Information about lead and PAHs concentrations in foodstuffs and their dietary intake is very important in human health risk assessment [12].

Currently, there are paucity of data on the risk assessment and concentrations of lead and polycyclic aromatic hydrocarbons in honey in Nigeria. This study has evaluated the human health risk assessment of lead and polycyclic aromatic hydrocarbons contaminations in honey from rural and urban communities in Enugu state, Nigeria.

\section{MATERIALS AND METHODS}

Twenty-five honey samples, $(1.50 \mathrm{~g})$ were collected from two rural sites considered (Enugu-Ezike and UzoUwani) and three urban sites considered (Opi-Nsukka, Obollo and $9^{\text {th }}$ miles) in Enugu state, Nigeria within the February and March 2016. These honey samples were extracted directly by squeezing the honey combs. Ten honey samples originated from rural communities while fifteen samples were from urban communities. The honey samples were collected in amber coloured glass bottles and stored at $4{ }^{\circ} \mathrm{C}$ prior to analysis.

\section{Digestion of honey samples and led determination}

$0.50 \mathrm{~g}$ of honey from each sample was digested in twenty-five millilitres aqua regia $\left(1: 370 \% \mathrm{HNO}_{3}\right.$ and pure $37 \%$ hydrochloric acid $(\mathrm{HCl})$ ) and allowed to cool to room temperature. It was filtered through a Whatman no. 1 filter paper and made up to 100 $\mathrm{ml}$ in volumetric flasks with deionized water before analyses. Three blanks were prepared by following the entire analytical procedure but omitting the samples.

The concentrations of $\mathrm{Pb}$ in honey samples were analysed in triplicates using an Atomic Absorption Spectrophotometer (Perkin Elmer AAS-700). The limit of detection (LOD) for $\mathrm{Pb}$ was $0.01 \mathrm{ppm}$, with blank values reading as $0.00 \mathrm{ppm}$ for deionized water with electrical conductivity value of lower than $5 \mu \mathrm{S} /$ $\mathrm{cm}$. Replicate analyses of several samples indicate the range of error to be $\pm 2 \%$ for $\mathrm{Pb}$ in the honey samples.

\section{Extraction and clean-up of PAHs}

Extraction and assay of PAHs were done by a modification of our previous method [12, 13, 14]. Briefly glass wares were washed thoroughly with hot detergent solution followed by rinsing with purified water and acetone (analytical grade) respectively. These were finally baked in the oven at $100^{\circ} \mathrm{C}$ overnight. The extraction of PAHs from the $0.50 \mathrm{~g}$ honey samples was done using a mixture of acetone and methylene chloride (50:50 ratio) spiked with $1 \mathrm{ml}$ of PAH internal standard and shaken thoroughly for proper mixing before placing in an ultrasonic bath. The concentrations of $16 \mathrm{PAH}$ (naphthalene, acenaphtylene, acenaphtene, fluorene, phenanthrene, anthracene, fluoranthene, pyrene, benzo[a]anthracene, chrysene, benzo[b]fluoranthene, benzo[k]fluoranthene, benzo[a]pyrene, indeno[1,2,3$\mathrm{c}, \mathrm{d}]$ pyrene, dibenzo[a,h]anthracene, and benzo[g,h,i] perylene) were analysed by gas chromatography (Gas Chromatograph - GC-FID) with GC recorder interfaced with a HP $[12,13,14]$. The EPA-16 PAHs determination was conducted at Jaros Inspection Services Limited, Port Harcourt, Rivers State, Nigeria using Gas Chromatographic System (6890 series and 6890 plus) equipped with a dual detector (FID-ECD), dual column and TriPlus AS auto-sampler with helium carrier gas based on the United State Environmental Protection Agency (US EPA) method 8100 (EPA 1984). A $2.00 \mu \mathrm{l}$ of extracts were injected into the GC port set at column conditions: HP-5 crosslinked PH-ME siloxane, length of $30 \mathrm{~m}$, I.D: $0.25 \mathrm{~mm}$, thickness of $1 \mu \mathrm{m}$ with helium carrier gas set in the spitless, constant flow mode with $1.2 \mathrm{ml} / \mathrm{min}$ flow rate. Other GC operating set-up were done according to the instrument's method development as specified in the operating instruction manual. Identification and quantification of individual PAHs was based on internal calibration standard containing known concentrations of the 16 PAHs (EPA-16). The specificity of the 16 PAHs sought for in the samples was confirmed by the presence of transition ions (quantifier and qualifier) as shown by their retention times which corresponded to those of their respective standards. The measured peak area ratios of precursor to quantifier ion were in close agreement with those of the standards.

The detection limit (LOD), estimated as three times the background noise (IUPAC criterion), was similar for all analysed compounds and results were less than $0.015 \mu \mathrm{g} / \mathrm{kg}$ d.w. (dry weight) for all analytes. The blank values of analytical procedure remained always below the quantification limit (LOQ): $0.05 \mu \mathrm{g} / \mathrm{kg}$ d.w., estimated as 10 times [12, 13, 14].

\section{Quality control and quality assurance}

All solvents used (e.g. dichloromethane) were pico grade quality. External calibrations were obtained with PAH solutions at five concentration levels. To evaluate 
the extraction efficiency for the target compounds, recovery studies were done by introducing known concentrations of standard PAH mixtures added to selected analyzed samples and the entire analytical steps from extraction to clean up were repeated. The percentage recovery was $95 \%$ of the standard. The matrix effect was evaluated by spiking the sample with a concentration range used for calibration and then comparing the correlation coefficient and slope of the spiked standard to the matrix with the original standard calibration curve. No matrix effect was observed.

\section{Cancer risk assessment}

The carcinogenic risk was estimated by calculating the incremental probability that an individual will develop cancer over a lifetime because of chronic exposure to a substance (that is, above baseline lifetime risk).

\section{Non-cancer risk assessment}

Calculation of daily intake of lead [15].

The Daily Intake of lead was calculated according

$$
D I M=\frac{C \times I R}{B W}
$$

Where:

DIM - Daily Intake of lead (mg/kg/day); C - lead concentration in honey; IR - honey intake; BW- average body weight for adult and for child: $70 \mathrm{~kg}$ and $24 \mathrm{~kg}$ respectively.

The THQ (Target Hazard Quotient) were calculated using the method below [18].

$$
T H Q=\frac{E f r \times E D \times I R \times C}{R f D o \times B W \times A T n} \times 10^{-3}
$$

Where:

Efr - exposure frequency (365 days/year); ED - exposure duration (58 years, equivalent to average life time of a Nigerian adult); IR - intake rate; C - concentration of metal; RfDo - oral reference dose; BW - average body weight of adult (70 kg); ATn - average exposure time for non-carcinogen in days (Efr x ED); $10^{-3}-$ conversion factor

The hazard index (HI) was used to estimate total chronic-toxic risks of multiple metals on the assumption of dose additivity [19]. In this study, the average daily intake of honey and among adults and children as obtained through questionnaire-based survey study are $8.4 \mathrm{~g} /$ day and $4.2 \mathrm{~g} /$ day respectively [20].

\section{Cancer Risks}

Incremental lifetime cancer risk (ILCR) is obtained using the Cancer Slope Factor (CSF), which is the risk produced by a lifetime average dose of $1 \mathrm{mg} / \mathrm{kg} \mathrm{bw}$ day $^{-1}$ and is contaminant specific.

$$
I L C R=C D I \times C S F
$$

Where:

$\mathrm{CDI}$ - chronic daily intake of chemical, $\mathrm{mg} / \mathrm{kg}$ bw day ${ }^{-1}$ represents the lifetime average daily dose exposure to the chemical.

$$
C D I=\frac{E D I \times E F r \times E D}{A t n}
$$

Where:

EDI - estimated daily intake of metal via consumption of honey; $\mathrm{EFr}$ - exposure frequency (365 days/year); ED - exposure duration 58 years; ATn - average exposure time for non-carcinogen in days (Efr x ED) [21].

The total cancer risk because of exposure to multiple contaminants due to consumption of a particular honey sample will be assumed to be the sum of the individual metal incremental risks.

\section{Benzo[a]pyrene equivalent (B[a]Peq) estimation}

Since benzo[a]pyrene is considered the most potent carcinogenic $\mathrm{PAH}$ the total $\mathrm{PAH}$ concentration is expressed as $\mathrm{B}[\mathrm{a}] \mathrm{P}$ equivalents $(\mathrm{B}[\mathrm{a}] \mathrm{Peq})$ to illustrate the toxic potency [22]. The B[a]Peq was calculated as the sum of the B[a]Peqi values for individual PAHs. The B[a]Peqi value was calculated for each PAH from its concentration in the sample (cPAHi) multiplied by its toxic equivalency factor (TEFPAHi) [23].

$$
B[a] P e q=\sum(B a P e q i)=\sum(P A H i+P A H i)
$$

\section{Margin of exposure}

The MOE is the ratio of the Benchmark-dose lower limit (BMDL10) to the estimated human intake of the compound. The BMDL10 is the lower bound of a $95 \%$ confidence interval on the Benchmark Dose corresponding to a $10 \%$ tumour incidence. This was chosen as a reference point in the dose-response curve $[24,25]$.

\section{Statistical Analysis}

Data analysis was done using SPSS statistical package (SPSS-20). Student's t-test was performed to compare lead levels in different honey samples. Difference between means was obtained using the least significance difference (LSD) at $p<0.05$. 


\section{RESULTS}

\section{Lead concentrations in samples of honey}

Table 1 shows the concentrations of lead $(\mathrm{Pb})$ in samples of honey collected from the two different rural sites and three different urban sites in Enugu. The mean concentration of lead in honey ranged from $0.01-0.081 \mathrm{mg} / \mathrm{kg}$. The highest level of $\mathrm{Pb}(0.081 \mathrm{mg} /$ $\mathrm{kg}$ ) contaminations was detected in honey sampled from Opi-Nsukka while the least level was detected in Uzo-Uwani.

\section{Human health risk assessment}

\section{Heavy metal - lead}

The estimated daily intake (EDI) of lead for adult (AD) and children $(\mathrm{CH})$ population via consumption of honey harvested in Enugu-Ezike, Uzo-Uwani, OpiNsukka, Obollo and $9^{\text {th }}$ miles are shown in Table 2. The EDIs of $\mathrm{Pb}$ for adult and children were in the range of (5.88E-07-9.72E-06) and (8.58E-07- 1.42E05 ) in $\mathrm{mg} / \mathrm{kg}$ bw day ${ }^{-1}$ respectively.

Table 1. Concentrations of lead $(\mathrm{mg} / \mathrm{kg})$ in honey in rural and urban areas

\begin{tabular}{|c|c|c|c|c|}
\hline \multicolumn{3}{|c|}{ Sample site } \\
\hline \multicolumn{2}{|c|}{ Rural } & \multicolumn{3}{c|}{ Urban } \\
\hline Enugu-Ezike & Uzo-Uwani & Opi-Nsukka & Obollo & $9^{\text {th } M i l e ~}$ \\
\hline $0.007 \pm 0.00^{\mathrm{a}}$ & $0.005 \pm 0.00^{\mathrm{a}}$ & $0.081 \pm 0.01^{\mathrm{b}}$ & $0.06 \pm 0.01^{\mathrm{b}}$ & $0.04 \pm 0.03^{\mathrm{c}}$ \\
\hline
\end{tabular}

Note: letters indicate that differences within groups (Rural and Urban sampling sites) were statistically significant.

Table 2. Estimated daily intake (EDI) of lead in honey samples among adults and children

\begin{tabular}{|c|c|c|c|c|c|c|c|c|c|}
\hline \multicolumn{9}{|c|}{ Sample site } \\
\hline \multicolumn{3}{|c|}{ Rural } & \multicolumn{2}{c|}{ Urban } \\
\hline \multicolumn{2}{|c|}{ Enugu-Ezike } & Uzo-Uwani & Opi-Nsukka & \multicolumn{2}{c|}{ Obollo } & \multicolumn{2}{c|}{9 Mile } \\
\hline $\mathrm{AD}$ & $\mathrm{CH}$ & $\mathrm{AD}$ & $\mathrm{CH}$ & $\mathrm{AD}$ & $\mathrm{CH}$ & $\mathrm{AD}$ & $\mathrm{CH}$ & $\mathrm{AD}$ & $\mathrm{CH}$ \\
\hline $2.1 \mathrm{E}-04$ & $3.06 \mathrm{E}-04$ & $1.50 \mathrm{E}-04$ & $2.1 \mathrm{E}-04$ & $2.43 \mathrm{E}-03$ & $3.54 \mathrm{E}-03$ & $1.8 \mathrm{E}-03$ & $2.63 \mathrm{E}-03$ & $1.2 \mathrm{E}-03$ & $1.75 \mathrm{E}-03$ \\
\hline
\end{tabular}

$\mathrm{AD}$ - adults; $\mathrm{CH}$ - children

Table 3 shows Target Hazard Quotient THQ of lead in honey samples from rural (Enugu-Ezike and Uzo-Uwani) and urban (Opi-Nsukka, Obollo and $9^{\text {th }}$ Mile) communities among adults and children. The THQs of $\mathrm{Pb}$ are at the range of $(1.50 \mathrm{E}-04-2.43 \mathrm{E}-$ 03 ) and (3.06E-04- 3.54E-03) for adults and children respectively. All the THQs were less than 1.
The incremental life time cancer risk (ILCR) of lead arising from consumption of honey harvested from rural (Enugu-Ezike and Uzo-Uwani) and urban (OpiNsukka, Obollo and $9^{\text {th }}$ Mile) communities among adults and children is shown in Table 4. The cancer risk ranged from 1.79E-06 - 2.07E-05 and 8.60E-073.01E-05 for adults and children respectively.

Table 3. Target Hazard quotient THQ of Lead in honey samples among adults and children

\begin{tabular}{|c|c|c|c|c|c|c|c|c|c|}
\hline \multicolumn{9}{|c|}{ Sample site } \\
\hline \multicolumn{9}{|c|}{ Rural } & \multicolumn{9}{c|}{ Urban } \\
\hline $\mathrm{AD}$ & $\mathrm{CH}$ & $\mathrm{AD}$ & $\mathrm{CH}$ & $\mathrm{AD}$ & $\mathrm{CH}$ & $\mathrm{AD}$ & $\mathrm{CH}$ & $\mathrm{AD}$ & $\mathrm{CH}$ \\
\hline $8.40 \mathrm{E}-07$ & $1.23 \mathrm{E}-06$ & $5.88 \mathrm{E} 7$ & $8.58 \mathrm{E} 07$ & $9.6 \mathrm{E}-06$ & $1.42 \mathrm{E}-05$ & $7.20 \mathrm{E}-06$ & $1.05 \mathrm{E}-05$ & $4.80 \mathrm{E}-06$ & $7.00 \mathrm{E}-06$ \\
\hline
\end{tabular}

$\mathrm{AD}$ - adults; $\mathrm{CH}$ - children

Table 4. ILCR for adults and children exposed to lead in honey

\begin{tabular}{|c|c|c|c|c|c|c|c|c|c|}
\hline \multicolumn{3}{|c|}{ Sample site } \\
\hline \multicolumn{3}{|c|}{ Rural } & \multicolumn{2}{c|}{ Urban } \\
\hline \multicolumn{2}{|c|}{ Enugu-Ezike } & Uzo-Uwani & \multicolumn{2}{c|}{ Opi-Nsukka } & \multicolumn{2}{c|}{ Obollo } & \multicolumn{2}{c|}{$9^{\text {th } M i l e ~}$} \\
\hline $\mathrm{AD}$ & $\mathrm{CH}$ & $\mathrm{AD}$ & $\mathrm{CH}$ & $\mathrm{AD}$ & $\mathrm{CH}$ & $\mathrm{AD}$ & $\mathrm{CH}$ & $\mathrm{AD}$ & $\mathrm{CH}$ \\
\hline $1.79 \mathrm{E}-06$ & $2.60 \mathrm{E}-06$ & $5.10 \mathrm{E}-06$ & $8.60 \mathrm{E}-07$ & $2.07 \mathrm{E}-05$ & $3.01 \mathrm{E}-05$ & $1.53 \mathrm{E}-05$ & $2.24 \mathrm{E}-05$ & $1.02 \mathrm{E}-05$ & $1.49 \mathrm{E}-05$ \\
\hline
\end{tabular}

AD - Adults; CH - Children; ILCR - Incremental lifetime cancer risk 
Polycyclic aromatic hydrocarbons contamination of honey

The concentration of the 16 priority PAHs (naphthalene, acenaphthylene, acenaphthene, anthracene, phenanthrene, flourene, flouranthene, pyrene, benzo[a] anthracene, chrysene, benzo[a]pyrene, benzo[b]flouranthene, benzo[k]flouranthene, benzo[g,h,i]perylene, dibenz[a,h]anthracene and ideno[1,2,3-cd]pyrene) evaluated in this study ranged from (4.71E-03-2.72) $\mathrm{mg} / \mathrm{kg}$ as shown in Table 5. Acenapthylene (ACEN) was highest level $(2.7 \mathrm{mg} / \mathrm{kg})$ in honey sample from $9^{\text {th }}$ mile (Urban sampling site). The lowest PAH level was found in benzo[b]flouranthene $(4.71 \mathrm{E}-03 \mathrm{mg} / \mathrm{kg}$ ) seen in honey sample from Uzo-Uwani (Rural sampling site). The same trend was also observed in the results of total PAH ( $\left.\sum \mathrm{PAH}\right)$. Benzo(b)fluoranthene and benz(a)anthracene were detected from honey in all the five sampled sites. B(a)P levels ranged from 2.27E$01-5.24 \mathrm{E}-01 \mathrm{mg} / \mathrm{kg}$ but was below the level of detection in Uzo-Uwani. The B[a]Peq ranged from 3.32E05 for pyrene in honey from Uzo-Uwani to $8.70 \mathrm{E} 01$ for dibenz(ah)anthracene in honey from Obollo. The $\Sigma \mathrm{B}$ [a]Peq ranged from 9.77E-02 to 1.31 in honey from Uzo-Uwani and Obollo respectively.

Table 5. Concentration of individual PAHs $(\mathrm{mg} / \mathrm{kg})$ and PAHs BaPeq $(\mathrm{mg} / \mathrm{kg})$ in honey

\begin{tabular}{|c|c|c|c|c|c|}
\hline \multirow{3}{*}{$\begin{array}{l}\text { Individual } \\
\text { PAHs }\end{array}$} & \multicolumn{5}{|c|}{ Sample sites } \\
\hline & \multicolumn{2}{|c|}{ Rural } & \multicolumn{3}{|c|}{ Urban } \\
\hline & $\mathrm{EE}$ & UU & $\mathrm{OP}$ & OB & $9 \mathrm{M}$ \\
\hline NAP & ND & ND & $\begin{array}{c}3.40 \mathrm{E}-01 \pm 0.00 \\
3.40 \mathrm{E}-04^{*}\end{array}$ & $\begin{array}{c}4.24 \mathrm{E}-01 \pm 0.00 \\
4.24 \mathrm{E}-4 *\end{array}$ & $\begin{array}{l}1.34 \pm 0.00 \\
\text { 1.34E-3* }\end{array}$ \\
\hline $\mathrm{ACE}$ & ND & ND & $\begin{array}{c}6.61 \mathrm{E}-01 \pm 0.00 \\
6.61 \mathrm{E}-4^{*}\end{array}$ & $\begin{array}{c}3.58 \mathrm{E}-01 \pm 0.00 \\
3.58 \mathrm{E}-4 *\end{array}$ & $\begin{array}{l}1.82 \pm 1.63 \\
\mathbf{1 . 8 2 E}-3^{*}\end{array}$ \\
\hline ACEN & ND & ND & $\begin{array}{c}4.40 \mathrm{E}-01 \pm 0.00 \\
4.40 \mathrm{E}-4^{*}\end{array}$ & $\begin{array}{c}1.61 \mathrm{E}-01 \pm 0.00 \\
1.61 \mathrm{E}-4 *\end{array}$ & $\begin{array}{l}2.72 \pm 2.38 \\
\mathbf{2 . 7 2 E - 3 *}\end{array}$ \\
\hline FLR & $\begin{array}{l}5.96 \mathrm{E}-02 \\
5.96 \mathrm{E}-5^{*}\end{array}$ & $\begin{array}{c}2.24 \mathrm{E}-02 \pm 0.00 \\
2.24 \mathrm{E}-5^{*}\end{array}$ & $\begin{array}{c}4.38 \mathrm{E}-01 \pm 0.00 \\
4.38 \mathrm{E}-4^{*}\end{array}$ & $\begin{array}{c}4.66 \mathrm{E}-01 \pm 0.29 \\
4.66 \mathrm{E}-4^{*}\end{array}$ & ND \\
\hline PHN & ND & ND & $\begin{array}{c}2.78 \mathrm{E}-01 \pm 0.00 \\
2.78 \mathrm{E}-4^{*}\end{array}$ & $\begin{array}{c}1.77 \mathrm{E}-01 \pm 0.00 \\
1.77 \mathrm{E}-4^{*}\end{array}$ & $\begin{array}{c}0.278 \pm 0.00 \\
2.78 \mathrm{E}-4^{*}\end{array}$ \\
\hline ANT & $\begin{array}{l}4.25 \mathrm{E}-02 \\
2.8 \mathrm{E}-4^{*}\end{array}$ & ND & $\begin{array}{c}5.99 \mathrm{E}-01 \pm 0.50 \\
5.9 \mathrm{E}-3 *\end{array}$ & $\begin{array}{c}6.50 \mathrm{E}-01 \pm 0.00 \\
6.5 \mathrm{E}-3^{*}\end{array}$ & ND \\
\hline FLT & ND & $\begin{array}{c}1.72 \mathrm{E}-02 \pm 0.00 \\
1.72 \mathrm{E}-5 *\end{array}$ & $\begin{array}{c}4.49 \mathrm{E}-01 \pm 0.33 \\
4.49 \mathrm{E}-4^{*}\end{array}$ & $\begin{array}{c}6.25 \mathrm{E}-01 \pm 0.45 \\
6.25 \mathrm{E}-4^{*}\end{array}$ & $\begin{array}{c}0.62 \pm 0.00 \\
6.20 \mathrm{E}-4^{*}\end{array}$ \\
\hline PYR & ND & $\begin{array}{c}3.32 \mathrm{E}-02 \pm 0.00 \\
\text { 3.3E-5* }\end{array}$ & $\begin{array}{c}4.64 \mathrm{E}-01 \pm 0.39 \\
4.6 \mathrm{E}-4 *\end{array}$ & $\begin{array}{c}5.16 \mathrm{E}-01 \pm 0.39 \\
5.2 \mathrm{E}-4 *\end{array}$ & ND \\
\hline BAA & $\begin{array}{l}\text { 7.30E-02 } \\
7.30 \mathrm{E}-3 *\end{array}$ & $\begin{array}{c}1.89 \mathrm{E}-02 \pm 0.00 \\
1.89 \mathrm{E}-3 *\end{array}$ & $\begin{array}{c}3.24 \mathrm{E}-01 \pm 0.26 \\
3.24 \mathrm{E}-2 *\end{array}$ & $\begin{array}{c}6.85 \mathrm{E}-01 \pm 0.29 \\
6.85 \mathrm{E}-2^{*}\end{array}$ & $\begin{array}{l}1.09 \pm 1.19 \\
\mathbf{1 . 0 9 E}-1^{*}\end{array}$ \\
\hline $\mathrm{CHY}$ & ND & $\begin{array}{c}2.03 \mathrm{E}-02 \pm 0.00 \\
2.03 \mathrm{E}-5^{*}\end{array}$ & $\begin{array}{c}3.33 \mathrm{E}-01 \pm 0.41 \\
3.33 \mathrm{E}-3 *\end{array}$ & $\begin{array}{c}7.43 \mathrm{E}-01 \pm 0.00 \\
7.43 \mathrm{E}-4^{*}\end{array}$ & $\begin{array}{l}1.865 \pm 1.18 \\
\mathbf{1 . 8 7 E - 0 3 *}\end{array}$ \\
\hline $\mathrm{BBF}$ & $\begin{array}{l}2.58 \mathrm{E}-01 \\
2.58 \mathrm{E}-2 *\end{array}$ & $\begin{array}{c}4.71 \mathrm{E}-03 \pm 0.00 \\
4.71 \mathrm{E}-4^{*}\end{array}$ & $\begin{array}{c}5.53 \mathrm{E}-01 \pm 0.62 \\
5.53 \mathrm{E}-2 *\end{array}$ & $\begin{array}{c}8.08 \mathrm{E}-02 \pm 0.00 \\
8.08 \mathrm{E}-3^{*}\end{array}$ & $\begin{array}{c}1.60 \pm 0.001 \\
1.60 \mathrm{E}-2 *\end{array}$ \\
\hline $\mathrm{BKF}$ & $\begin{array}{l}7.86 \mathrm{E}-02 \\
7.86 \mathrm{E}-3^{*}\end{array}$ & ND & $\begin{array}{c}6.35 \mathrm{E}-02 \pm 0.01 \\
6.35 \mathrm{E}-3^{*}\end{array}$ & ND & $\begin{array}{l}1.23 \pm 0.00 \\
\mathbf{1 . 2 3 E}-1 *\end{array}$ \\
\hline BAP & $\begin{array}{c}2.27 \mathrm{E}-01 \\
2.3 \mathrm{E}-1 *\end{array}$ & ND & $\begin{array}{c}5.24 \mathrm{E}-01 \pm 0.43 \\
5.24 \mathrm{E}-*\end{array}$ & $\begin{array}{c}2.99 \mathrm{E}-01 \pm 0.20 \\
2.9 \mathrm{E}-1^{*}\end{array}$ & $\begin{array}{c}0.1 \pm 0.00 \\
0.1 *\end{array}$ \\
\hline $\mathrm{B}$ (ghi)P & $\begin{array}{l}1.49 \mathrm{E}-01 \\
1.49 \mathrm{E}-3 *\end{array}$ & ND & $\begin{array}{c}4.35 \mathrm{E}-01 \pm 0.39 \\
4.35 \mathrm{E}-3^{*}\end{array}$ & ND & $\begin{array}{c}0.12 \pm 0.00 \\
\text { 1.20E-3* }\end{array}$ \\
\hline DBA & $\begin{array}{l}1.73 \mathrm{E}-01 \\
1.73 \mathrm{E}-1 *\end{array}$ & $\begin{array}{c}9.48 \mathrm{E}-02 \pm 0.00 \\
9.48 \mathrm{E}-2 *\end{array}$ & $\begin{array}{c}4.71 \mathrm{E}-01 \pm 0.39 \\
4.71 \mathrm{E}-1 *\end{array}$ & $\begin{array}{c}8.70 \mathrm{E}-01 \pm 0.00 \\
8.70 \mathrm{E}-1 *\end{array}$ & ND \\
\hline INP & $\begin{array}{l}2.42 \mathrm{E}-01 \\
2.42 \mathrm{E}-2 *\end{array}$ & ND & $\begin{array}{c}4.89 \mathrm{E}-01 \pm 0.26 \\
4.89 \mathrm{E}-2 *\end{array}$ & $\begin{array}{c}5.53 \mathrm{E}-01 \pm 0.00 \\
5.53 \mathrm{E}-2 *\end{array}$ & $\begin{array}{c}0.51 \pm 0.00 \\
\text { 5.10E-2* }\end{array}$ \\
\hline$\sum \mathrm{PAH}$ & $\begin{array}{c}1.30 \mathrm{E} \pm 0.00 \\
4.67 \mathrm{E}-1^{*}\end{array}$ & $\begin{array}{l}2.51 \mathrm{E}-01 \\
\text { 9.77E-2* }\end{array}$ & $\begin{array}{c}3.07 \pm 2.10 \\
1.15^{*}\end{array}$ & $\begin{array}{c}1.86 \pm 2.58 \\
1.31 *\end{array}$ & $\begin{array}{c}3.08 \pm 1.89 \\
\text { 4.09E-1* }\end{array}$ \\
\hline
\end{tabular}

Note: BBF - benzo(b)fluoranthene, BKF - benzo(k)fluoranthene, BAP- benzo(a)pyrene, B(ghi)P -benzo(g,h,i)perylene, DBA - BaPeq of PAHs values are presented in exterics. NAP - naphthalene, ACEN-acenapthylene, ACE - acenaphthene, FLR - fuorene, PHN - phenanthrene, ANT - anthracene, FLT - fluoranthene, PYR- Pyrene, BAA - benz(a)anthracene, CHY - chrysene, BBF-dibenz(ah) anthracene, INP - indeno(1,2,3-cd)pyrene, ND - not detected. 
The concentration of $\mathrm{B}(\mathrm{a}) \mathrm{P}, \sum \mathrm{PAH} 16, \mathrm{PAH} 4$ PAH8 and $\sum$ B(a)Peq ranged from (0.1-0.524) $\mathrm{mg} / \mathrm{kg}$ $(0.251-3.08) \mathrm{mg} / \mathrm{kg}(0.0439-3.22) \mathrm{mg} / \mathrm{kg},(0.0439-$ $3.32) \mathrm{mg} / \mathrm{kg}$ and $(0.0977-1.31) \mathrm{mg} / \mathrm{kg}$ respectively as shown in Table 6 . The concentration of $\mathrm{B}(\mathrm{a}) \mathrm{P}$ in honey samples ranged from $(0.1-0.524) \mathrm{mg} / \mathrm{kg}$, honey sample from Uzo-Uwani had no detectable level of $\mathrm{B}(\mathrm{a}) \mathrm{P}$, with highest $\mathrm{B}(\mathrm{a}) \mathrm{P}$ detected in Opi Nsukka $(0.524 \mathrm{mg} / \mathrm{kg})$. The mean concentration of the $\sum$ PAHs in honey samples ranged from $(0.251$ $3.08 \mathrm{mg} / \mathrm{kg}$ ) $\mathrm{mg} / \mathrm{kg}$. The highest level of $\sum$ PAH was detected in honey sampled from $9^{\text {th }}$ miles $(3.08 \mathrm{mg} /$ $\mathrm{kg}$ ) while the lowest was detected in honey from Uzo-Uwani $(0.251 \mathrm{mg} / \mathrm{kg})$.

Table 6. B(a)P, $\sum$ PAH, PAH4, PAH8 concentrations ( $\mathrm{mg} / \mathrm{kg}$ ) and $\sum \mathrm{B}(\mathrm{a}) \mathrm{Peq}$ in honey

\begin{tabular}{|c|c|c|c|c|c|c|}
\hline \multicolumn{2}{|c|}{ Sample sites } & $\mathrm{B}[\mathrm{a}] \mathrm{P}$ & $\sum \mathrm{PAH}$ & PAH4 & PAH8 & $\sum \mathrm{B}(\mathrm{a}) \mathrm{Peq}$ \\
\hline \multirow{2}{*}{ 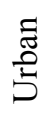 } & Enugu-Ezike & 0.524 & 3.07 & 1.73 & 2.25 & 1.15 \\
\hline & Uzo-Uwani & ND & 0.251 & 4.39E-02 & 4.39E-02 & $9.77 \mathrm{E}-02$ \\
\hline \multirow{3}{*}{ 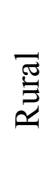 } & Opi Nsukka & 0.227 & 1.30 & $5.58 \mathrm{E}-01$ & 0.785 & 4.67E-01 \\
\hline & Obollo & 0.299 & 1.86 & 1.81 & 2.11 & 1.31 \\
\hline & $9^{\text {th }}$ Mile & 0.1 & 3.08 & 3.22 & 3.32 & $4.09 \mathrm{E}-01$ \\
\hline
\end{tabular}

PAH8 - genotoxic 8 PAHs include the sum of benz[a]anthracene, chrysene, benzo[b]fluoranthene, benzo[k]fluoranthene, benzo[a]pyrene, indeno[1,2,3-cd]pyrene, dibenz[a,h]anthracene, and benzo[ghi]perylene; PAH4 - benz[a]anthracene, chrysene, benzo[a]pyrene, benzo[b] fluoranthene $\mathrm{B}[\mathrm{a}] \mathrm{Peq}$ - benzo[a]pyrene equivalent estimation.

The PAH4, PAH8 and $\sum \mathrm{B}(\mathrm{a})$ Peq levels range of $(0.0439-3.22) \mathrm{mg} / \mathrm{kg},(0.0439-3.32) \mathrm{mg} / \mathrm{kg}$ and $(0.0977-1.31) \mathrm{mg} / \mathrm{kg}$ respectively. Highest level of PAH4 was detected in honey sampled from $9^{\text {th }}$ mile $(3.22 \mathrm{mg} / \mathrm{kg})$ while the lowest was in Uzo-Uwani $(0.0439 \mathrm{mg} / \mathrm{kg})$. Highest level of PAH8was detected in honey sampled from $9^{\text {th }}$ mile $(3.32 \mathrm{mg} / \mathrm{kg})$ while the lowest was in Uzo-Uwani $(0.0439 \mathrm{mg} / \mathrm{kg})$. The highest level of $\sum \mathrm{B}(\mathrm{a}) \mathrm{Peq}$ was detected in honey sampled from $9^{\text {th }}$ mile $(1.31 \mathrm{mg} / \mathrm{kg})$ while the lowest was in Uzo-Uwani $(0.0977 \mathrm{mg} / \mathrm{kg})$.
Table 7 shows the EDI of $\mathrm{B}(\mathrm{a}) \mathrm{P}, \sum \mathrm{PAH}, \mathrm{PAH}$, PAH8 and $\sum \mathrm{B}(\mathrm{a}) \mathrm{Peq}$ in honey among adults and children. The EDI $\mathrm{B}(\mathrm{a}) \mathrm{P}$ in adults and children ranged from $(8.40 \mathrm{E}-04-4.40 \mathrm{E}-03) \mathrm{mg} / \mathrm{kg} /$ day and $(4.20 \mathrm{E}-04-$ $2.20 \mathrm{E}-03) \mathrm{mg} / \mathrm{kg} /$ day respectively. The highest level of B(a)P intake for both adult and children was observed in the honey sampled from Opi-Nsukka (4.40E-03) mg/ $\mathrm{kg} /$ day for adult and (2.20E-03) $\mathrm{mg} / \mathrm{kg} /$ day for children while the lowest EDI value was observed in honey from $9^{\text {th }}$ mile $(8.40 \mathrm{E}-04 \mathrm{mg} / \mathrm{kg} / \mathrm{day})$ for adult and $(4.20 \mathrm{E}-04$ $\mathrm{mg} / \mathrm{kg} /$ day)for children. The concentration of $\mathrm{B}(\mathrm{a}) \mathrm{P}$ was below the limit of detection in Uzo-Uwani.

Table 7. Estimated daily intake (EDI) of PAHs group in honey $(\mathrm{mg} / \mathrm{kg} / \mathrm{day})$

\begin{tabular}{|c|c|c|c|c|c|c|c|c|c|c|c|}
\hline \multirow{2}{*}{\multicolumn{2}{|c|}{ Sample sites }} & \multicolumn{2}{|c|}{$\mathrm{B}[\mathrm{a}] \mathrm{P}$} & \multicolumn{2}{|c|}{$\sum \mathrm{PAH}$} & \multicolumn{2}{|c|}{ PAH4 } & \multicolumn{2}{|c|}{ PAH8 } & \multicolumn{2}{|c|}{$\sum \mathrm{B}(\mathrm{a}) \mathrm{Peq}$} \\
\hline & & $\mathrm{AD}$ & $\mathrm{CH}$ & $\mathrm{AD}$ & $\mathrm{CH}$ & $\mathrm{AD}$ & $\mathrm{CH}$ & $\mathrm{AD}$ & $\mathrm{CH}$ & $\mathrm{AD}$ & $\mathrm{CH}$ \\
\hline \multirow{2}{*}{ 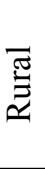 } & $\begin{array}{l}\text { Enugu } \\
\text { Ezike }\end{array}$ & $1.91 \mathrm{E}-03$ & $9.53 \mathrm{E}-04$ & 0.0109 & 0.0055 & 0.0047 & 0.0023 & 0.0066 & 0.0033 & $3.92 \mathrm{E}-03$ & $1.96 \mathrm{E}-03$ \\
\hline & $\begin{array}{c}\text { Uzo- } \\
\text { Uwani }\end{array}$ & ND & ND & 0.0021 & 0.0011 & 0.0004 & 0.0002 & 0.0004 & 0.0002 & $8.21 \mathrm{E}-04$ & $4.10 \mathrm{E}-04$ \\
\hline \multirow{3}{*}{ 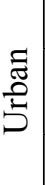 } & Opi & 4.40E-03 & $2.20 \mathrm{E}-03$ & 0.0258 & 0.0129 & 0.0145 & 0.0073 & 0.0189 & 0.0095 & $9.66 \mathrm{E}-03$ & $4.83 \mathrm{E}-03$ \\
\hline & $\begin{array}{l}\text { Nsukka } \\
\text { Obollo }\end{array}$ & $2.51 \mathrm{E}-03$ & $1.26 \mathrm{E}-03$ & 0.0156 & 0.0078 & 0.0152 & 0.0076 & 0.0177 & 0.0089 & $1.10 \mathrm{E}-02$ & $5.50 \mathrm{E}-03$ \\
\hline & $9^{\mathrm{TH}}$ Mile & 8.40E-04 & $4.20 \mathrm{E}-04$ & 0.0259 & 0.0129 & 0.0270 & 0.0135 & 0.0279 & 0.0139 & $3.44 \mathrm{E}-03$ & $1.72 \mathrm{E}-03$ \\
\hline
\end{tabular}

EDIPAH - $\Sigma \mathrm{i}$ PAHi, IR for children $(\mathrm{CH}) 24 \mathrm{~kg}=0.0042 \mathrm{~kg}$, IR for adult (AD) $70 \mathrm{~kg}=0.0084 \mathrm{~kg}$, ND- not detected, IR - ingestion rate.

The $\sum$ PAH daily intake among adults and children ranged from $(0.0021-0.0259) \mathrm{mg} / \mathrm{kg} /$ day and $(0.0011-$ $0.0129) \mathrm{mg} / \mathrm{kg} /$ day respectively. The highest EDI level of $\sum$ PAH for both adult and children was observed in the honey sampled in $9^{\text {th }}$ mile $(0.0259 \mathrm{mg} / \mathrm{kg} /$ day $)$ for adult and $(0.0129 \mathrm{mg} / \mathrm{kg} / \mathrm{day})$ for children while the lowest value was observed in Uzo-Uwani $(0.0021 \mathrm{mg} /$ $\mathrm{kg} /$ day $)$ for adult and $(0.0011 \mathrm{mg} / \mathrm{kg} /$ day $)$ for children.
PAH4 daily intake among adults and children ranged from $(0.0004-0.027) \mathrm{mg} / \mathrm{kg} /$ day and $(0.0002-0.0135)$ $\mathrm{mg} / \mathrm{kg} /$ day respectively. The highest EDI level of PAH4 for both adult and children was observed in the honey sampled from $9^{\text {th }}$ mile $(0.027 \mathrm{mg} / \mathrm{kg} /$ day) for adult and $(0.0135 \mathrm{mg} / \mathrm{kg} / \mathrm{day})$ for children while the lowest value was observed in Uzo-Uwani $(0.0004 \mathrm{mg} / \mathrm{kg} / \mathrm{day})$ for adult and $(0.0002 \mathrm{mg} / \mathrm{kg} /$ day $)$ 
for children. PAH8 daily intake among adults and children ranged from $(0.0004-0.0279) \mathrm{mg} / \mathrm{kg} / \mathrm{day}$ and (0.0002- 0.0139$) \mathrm{mg} / \mathrm{kg} /$ day respectively. The highest EDI level of PAH 8 for both adult and children was observed in the honey sampled at $9^{\text {th }}$ mile $(0.0279 \mathrm{mg} /$ $\mathrm{kg} /$ day) for adult and $(0.0139 \mathrm{mg} / \mathrm{kg} /$ day $)$ for children while the lowest value was detected in Uzo-Uwani $(0.0004 \mathrm{mg} / \mathrm{kg} /$ day $)$ for adult and $(0.0002 \mathrm{mg} / \mathrm{kg} /$ day $)$ for children. $\sum \mathrm{B}(\mathrm{a})$ Peq daily intake among adults and children ranged from $(8.21 \mathrm{E}-04-9.66 \mathrm{E}-03) \mathrm{mg} / \mathrm{kg} /$ day and (4.10E-04- 5.50E-03) mg/kg/day respectively. The highest EDI level of $\sum \mathrm{B}(\mathrm{a}) \mathrm{Peq}$ for both adult and children was observed in the honey sampled at Obollo $(9.66 \mathrm{E}-03 \mathrm{mg} / \mathrm{kg} /$ day) for adult and $(5.50 \mathrm{E}-03 \mathrm{mg} / \mathrm{kg} /$ day) for children while the lowest value was observed in Uzo-Uwani $(8.21 \mathrm{E}-04 \mathrm{mg} / \mathrm{kg} /$ day) for adult and (4.10E-04 mg/kg/day) for children.
Table 8 is the margin of exposure (MOE) and ILCR of $\mathrm{B}$ (a) $\mathrm{P}$ of PAHs groups in honey for adults and children. The MOE of $\mathrm{B}[\mathrm{a}] \mathrm{P}$ ranged from $1.59 \mathrm{E}+07$ $-8.33 \mathrm{E}+07$ for adult and children respectively, PAH 4 ranged from $1.26 \mathrm{E}+07-8.50 \mathrm{E}+08$ and $2.52 \mathrm{E}+07$ - $1.70 \mathrm{E}+10$ for adult and children respectively and PAH8 ranged from $1.76 \mathrm{E}+07-1.23 \mathrm{E}+10$ and 3.53 $\mathrm{E}+07-2.45 \mathrm{E}+10$ for adult and children respectively. The ILCR of $\mathrm{B}(\mathrm{a}) \mathrm{P}$ among adults and children ranged from $(8.5619 \mathrm{E}-11-1.1471 \mathrm{E}-09)$ and $(1.25 \mathrm{E}-10-1.67 \mathrm{E}-$ 09) respectively. The highest level of $\mathrm{B}(\mathrm{a}) \mathrm{P}$ for both adult and children was detected in the honey sampled from Obollo (1.1471E-09) for adult and (1.67E-09) for children while the lowest value was detected in Uzo-Uwani (8.40E-04) for adult and (1.25E-10) for children.

Table 8. Margin of Exposure (MOE) of PAHs groups and Incremental Lifetime Cancer Risk (ILCR) of B[a]P

\begin{tabular}{|c|c|c|c|c|c|c|c|c|c|}
\hline \multicolumn{2}{|c|}{ Sample sites } & \multicolumn{2}{|c|}{$\mathrm{B}[\mathrm{a}] \mathrm{P}$} & \multicolumn{2}{|c|}{ PAH4 } & \multicolumn{2}{|c|}{ PAH8 } & \multicolumn{2}{|c|}{ ILCR of $B[a] P$} \\
\hline \multirow{3}{*}{$\underset{\overparen{Z}}{\overparen{\pi}}$} & \multirow{2}{*}{$\begin{array}{l}\text { Enugu- } \\
\text { Ezike }\end{array}$} & $\mathrm{AD}$ & $\mathrm{CH}$ & $\mathrm{AD}$ & $\mathrm{CH}$ & $\mathrm{AD}$ & $\mathrm{CH}$ & $\mathrm{AD}$ & $\mathrm{CH}$ \\
\hline & & $3.66 \mathrm{E}+07$ & $7.35 \mathrm{E}+07$ & $7.2 \mathrm{E}+07$ & $1.48 \mathrm{E}+08$ & $7.42 \mathrm{E}+07$ & $1.48 \mathrm{E}+08$ & $4.088 \mathrm{E}-10$ & $4.088 \mathrm{E}-10$ \\
\hline & Uzo- Uwani & ND & ND & $8.50 \mathrm{E}+08$ & $1.70 \mathrm{E}+10$ & $1.23 \mathrm{E}+10$ & $2.45 \mathrm{E}+10$ & $8.5619 \mathrm{E}-11$ & $8.5619 \mathrm{E}-11$ \\
\hline \multirow{3}{*}{ 淽 } & Opi-Nsukka & $1.59 \mathrm{E}+07$ & $3.18 \mathrm{E}+07$ & $2.34 \mathrm{E}+07$ & $2.59 \mathrm{E}+07$ & $2.59 \mathrm{E}+07$ & $5.16 \mathrm{E}+07$ & $1.0074 \mathrm{E}-09$ & $1.0074 \mathrm{E}-09$ \\
\hline & Obollo & $2.79 \mathrm{E}+07$ & $5.56 \mathrm{E}+07$ & $2.24 \mathrm{E}+07$ & $2.77 \mathrm{E}+07$ & $2.77 \mathrm{E}+07$ & $5.51 \mathrm{E}+07$ & $1.1471 \mathrm{E}-09$ & $1.1471 \mathrm{E}-09$ \\
\hline & $9^{\text {th }}$ Mile & $8.33 \mathrm{E}+07$ & $1.67 \mathrm{E}+08$ & $1.26 \mathrm{E}+07$ & $1.76 \mathrm{E}+07$ & $1.76 \mathrm{E}+07$ & $3.53 \mathrm{E}+07$ & $3.5874 \mathrm{E}-10$ & $3.5874 \mathrm{E}-10$ \\
\hline
\end{tabular}

$\mathrm{CH}$ - children $(24 \mathrm{~kg}), \mathrm{AD}$ - adults $(70 \mathrm{~kg})$, ILCR - Incremental lifetime cancer risk.

\section{DISCUSSION}

Honey is such a highly valued natural meal in many parts of the world especially in Nigeria due to several health benefits associated with it. Lead and polycyclic aromatic hydrocarbons [26]. The concentrations of $\mathrm{Pb}$ in our study were lower than concentrations in previous studies $[27,28]$ where $\mathrm{Pb}$ levels were reported $\mathrm{Pb}$ levels $(<0.50-10.02 \mathrm{mg} / \mathrm{kg}$ ) and $10.2 \mathrm{mg} / \mathrm{kg}$ respectively. Proximity of bee hives to vehicular emissions could contribute to high $\mathrm{Pb}$ levels in honey [27]. Health implication of $\mathrm{Pb}$ exposure to human and animal include immunotoxicity, nervous and cardiovascular toxicity, and even musculotoxicity $[29,30]$.

Since the main exposure to lead is through the diet consequently the information about the dietary intake is very important to assess the risk to human health $[18,31]$. Carcinogenic risk is an estimation of the probability of an individual contracting cancer over a lifetime estimated as 58 years (Nigerians). Risks values exceeding $1 \times 10^{-4}$ are regarded as intolerable, risks less than $1 \times 10^{-6}$ do not produce adverse health effects, and risks lying between $1 \times 10^{-4}$ and $1 \times 10^{-6}$ are generally said to be a safe range [32]. With both
THQ less 1 and ILCR less than $1 \times 10^{-6}$, consumption of honey from Enugu State Nigeria may be safe from any intolerable effect from lead.

PAHs can enter the food chain by deposition from air, or by deposition and transfer from soil and water [33]. In this study, honey samples from the five sampling communities showed presence of $43.8-100 \%$ of the total 16 PAHs. Food can be contaminated with PAHs from environmental sources, techniques in industrial food processing and from certain unhealthy home cooking practices [25].

The carcinogenic potency of benzo[a]pyrene (BAP) makes it the most widely studied of the PAHs [34]. B(a)P equivalents (BAPeq) gives a conversion of the concentrations of each targeted PAHs in the honey to the carcinogenic equivalent of benzo[a] pyrene while the total BAPeq is the summation of the contributions of individual PAH B(a)P equivalents in honey [35], using toxicity equivalent factors $[36,37$, 38]. Toxic equivalency factor for individual is a unique value for each $\mathrm{PAH}$ which is explored in calculation of specific PAH, B(a)Peq values. The European Food Safety Authority CONTAM Panel suggested the use of the margin of exposure (MOE) approach for risk assessment [25]. 
The values of B(a)P, ¿PAH16 and PAH4 obtained in this study were higher than the levels reported in rape honey [39]. Honey from urban sampling communities showed higher PAHs levels than samples from rural communities. The EDI of $\mathrm{B}(\mathrm{a}) \mathrm{P}$ was observed to be lower than the limit of detection in $20 \%$ of the samples. There were $60 \%$ and $40 \%$ violations in levels of $\mathrm{B}(\mathrm{a}) \mathrm{P}$ in our samples among adults and children respectively as compared to EU standard of $1 \mu \mathrm{g} / \mathrm{kg} /$ day.

In this study, all the honey samples have the MOEs of the PAHs higher than 1.0 E+04. This indicated that the consumption of honey from the region may not pose any adverse health effects. The least MOE values were observed in PAH4 (1.76 E+07). European Food Safety Authority (EFSA) Scientific Committee expressed the view that in general, a margin of exposure of 1.0 $\mathrm{E}+04$ and above, based on the 'Benchmark-dose lower bound' (BMDL) from animal study, would be of low concern with respect to public health [25]. Our study showed that all MOE values for both B(a)P, PAH 4, PAH 8 and PAH 16 among adult and children were above the critical limit of 1.0 E04 [25] indicating negligible public health risk concern.

Co-existence of both lead and PAHs in the present study may be of public significance given that exposures to mixtures may synergize or be additive thus leading to adverse health effects exceeding those noted upon exposures to a single pollutant $[40,41]$. Atmospheric deposition, flooding and sediment deposition, sewage sludge and application of fertilizers are the likely sources of metals [42]. Lead as a redox inactive metal show its toxic effect via bonding to sulfhydryl groups of proteins and depletion of glutathione. In metabolism of $\mathrm{B}[\mathrm{a}] \mathrm{P}$, free radicals are formed as a by-product. In addition, the reactive metabolites of $\mathrm{B}[\mathrm{a}] \mathrm{P}$ undergoes a redox cycle and generates reactive oxygen species [43]. Although low doses of a single metal may not cause health effects, when combined with any other contaminants may present increased health risk [41] and the US EPA recognize this as a key gap in metal risk assessment [42].

\section{CONCLUSIONS}

Taken together consumption of honey from Enugu State, Nigeria may be safe but biomonitoring of lead and PAHs is recommended in the local population.

\section{Conflict of interest}

The authors declare no conflict of interest.

Advances in knowledge: Information on harmful health effects of heavy metals and PAHs does not only seem to represent a context unique to Africa but data on exposures are quite scanty. There is therefore need for more focus on current and emerging environmental and chemical health risks in sub Saharan African research studies. This study is a contribution to address the knowledge gap.

Application to patient care: As the advocacy for physicians to be mindful of dietary risk factors in the aetiology of certain diseases, honey consumption in Enugu State, Nigeria can be rightly said to be devoid of lead and PAHs risks.

\section{REFERENCES}

1. IARC. Polynuclear aromatic hydrocarbons. Part 1. Chemical, environmental and experimental data. IARC Monogr. Eval. Carcinog. Risk Chem. Hum. 1983;3:1453.

2. Scientific Committee on Food. Opinion of the Scientific Committee on Food and the risk to human health of Polycyclic Aromatic Hydrocarbon in food. SCF/CS/ CNTM/PAH/29 Final 4 December 2002 [Online: https://ec.europa.eu/food/sites/food/files/safety/docs/ sci-com_scf_out153_en.pdf

3. Ikeda $\bar{M}$, Zhang $Z \bar{W}$, Shimbo S, Nakatsuka H, Moon CS, Matsuda-Inoguchi N, Higashikawa K.: Urban population exposure to lead and Cadmium in east and south east Asia. Sci Total Environ.2000; 249 (1-3): 373384.

4. Bede-Ojimadu O, Amadi CN Orisakwe OE.: Blood lead level in women of child-bearing age in subsaharan Africa: A systematic review. Front. Public Health, 19 December 2018; https://doi.org/10.3389/ fpubh.2018.00367.

5. Anyanwu B, Ezejiofor A, Igweze Z.. Orisakwe OE.: Heavy Metals Mixture Exposure and effects in Developing Nations: An Update. Toxics. 2018;6(4):65. Available from: http://www.mdpi.com.

6. Türkdogan MK, Kilicel F, Kara K, Tuncer I, Uygan I.: Heavy metals in soil, vegetables and fruits in the endemic upper gastrointestinal cancer region of Turkey. Environ Toxicol Pharmacol. 2003;13(30):175-179.

7. Ioannidou MD, Zachariadis GA, Anthemidis AN, Stratis $J A$.: Direct determination of toxic trace metals in honey and sugars using inductively coupled plasma atomic emission spectrometry. Talanta 2005;65: 92-97.

8. Bogdanov S, Jurendic T, Sieber R, Gallmann P.: Honey for Nutrition and Health: A Review. Am J Coll Nutr. 2008;27: 677-689.

9. Sitarz-Palczak E, Kalembkiewicz J, Galas D.: Evaluation of the content of selected heavy metals in samples of Polish honeys. J. Ecol. Eng. 2015;16(3).

10. Costanza R, d'Arge R, De Groot R, Farber S, Grasso M, Hannon B, Limburg K, Naeem S, O'neill RV, Paruelo $J$, Raskin $R G$. : The value of the world's ecosystem services and natural capital. Nature 1997;87(6630):253.

11. Zhan $X$. Liang $X$, Jiang $T, X u$ G.: Interaction of phenanthrene and potassium uptake by wheat roots: a mechanistic model. BMC Plant Biol. 2013;13(1): 1-9.

12. Udowelle NA, Igweze ZN, Asomugha RN, Orisakwe $O E$.: Health Risk Assessment and Dietary Exposure 
to Polycyclic Aromatic Hydrocarbons (PAHs), Lead and Cadmium from Bread Consumed in Nigeria. Rocz Panstw Zakl Hig.2017;68(3): 269-280.

13. Orisakwe OE, Mbagwu HO, Ukpai P, Udowelle NA.: Survey of polycyclic aromatic hydrocarbons and lead in Chinese teas sold in Nigeria: levels and health implications. Rocz Panstw Zakl Hig 2015;66(3): 225232.

14. Ekhator OC, Udowelle NA, Igbiri S, Asomugha RN, Igweze ZN, Orisakwe OE.: Safety Evaluation of Potential Toxic Metal Exposure from Street Food Consumed in Mid-West Nigeria. J. Environ. Public Health. January 2017;26;2:1-8. https://doi.org/10.1155/2017/8458057.

15. US-EPA IRIS United States, Environmental Protection Agency, Integrated Risk Information System. 2006. $<$ http://www.epa. gov/iris/subst $>$.

16. WHO. Environmental Health Criteria 237. Principles for evaluating health risks in children associated with exposure to chemicals. Geneva, Switzerland: World Health Organization, 2006. whqlibdoc.who.int/ publications/2006/924157237X_eng.pdf

17. Igbiri $S$, Udowelle NA, Ekhator OC, Asomugha RN, Igweze ZN, Orisakwe OE.: Edible Mushrooms from Niger Delta, Nigeria with Heavy Metal Levels of Public Health Concern: A Human Health Risk Assessment. Recent Pat Food Nutr Agric. 2018;9(1):31-41. Doi: 10. 2174/2212798409666171129173802

18. Singh A, Sharma RK, Agrawal M, Marshall FM.: Risk assessment of heavy metal toxicity through contaminated vegetables from waste water irrigated area of Varanasi. India. Trop Ecol. 2010;51: 375-387.

19. US Environmental Protection Agency (US EPA). Supplemental Guidance for Developing soil Screening Levels for Superfund Sites,. Office of Solid Waste and Emergency Response; Washington, DC, USA, OSWER, 2001;9355: 4-24.

20. Ozoani HA.: Health risk assessment of some metals and polycyclic aromatic hydrocarbons in honey and other environmental matrices in Enugu State, Nigeria. MSc. dissertation, University of Port Harcourt, Nigeria, 2018.

21. Bamuwamye M, Ogwok P, Tumuhairwe V.: Cancer and non-cancer risks associated with heavy metal exposures from street foods: Evaluation of roasted meats in an urban setting. J. of Environ Poll and Human Health. 2015;3(2): 24-30.

22. Perugini $M$, Visciano $P$, Giammarino A, Manera $M$, Di Nardo $W$, Amorena M.: Polycyclic aromatic hydrocarbons in marine organisms from the Adriatic Sea, Italy. Chemosphere 2007;66:1904-1910.

23. Nisbet ICT, LaGoy PK.: Toxic equivalency factors (TEFs) for polycyclic aromatic hydrocarbons (PAH). Regul Toxicol Pharmacol 1993;16: 290-300.

24. EFSA. Scientific Committee Scientific Opinion on the applicability of the margin of exposure approach for the safety assessment of impurities which are both genotoxic and carcinogenic in substances added to food/feed. EFSA J. 2012;10(3): 2578.

25. EFSA, Scientific opinion of the panel on contaminants in the food chain on a request from the European Commission on polycyclic aromatic hydrocarbons in food. EFSA J. 2008;724: 1-114.
26. D'iez S, Delgado S, Aguilera I.: Prenatal and early childhood exposure to mercury and methylmercury in Spain, a high-fish-consumer country. Arch. of Environ. Contam Toxicol. 2009; 56(3): 615-622.

27. Iwegbue CMA, Obi-Iyeke GE, Tesi GO, Obi G, Bassey FI.: Concentrations of selected metals in honey consumed in Nigeria. Int. J. Environ. Studies 2015;72(4) 713-722. DOI:10.1080/00207233.2015.102 8783

28. Altun SK, Dinç H, Paksoy N, Temamoğulları FK, Savrunlu M.: Analyses of Mineral Content and Heavy Metal of Honey Samples from South and East Region of Turkey by Using ICP-MS. Int. J. Anal. Chem. 2017; Article ID 6391454, 6 pages,. https://doi. org/10.1155/2017/6391454

29. Kalia K, Flora SJS.: Strategies for Safe and Effective Therapeutic Measures for Chronic Arsenic and Lead Poisoning. J. Occup. Health 2005;47:11-21. http:// dx.doi.org/10.1539/joh.47.1

30. Martinello $M$, Dainese $N$, Manzinello $C$. Borin A.: Retrospective evaluation of lead contamination in honey from 2005 to present in northeastern Italy and future perspectives. Food Addit Contam. Part B. Survieillance 2016;9(3):1939-3229.

31. Llobet JM, Domingo JL, Bocio A, Casas C, Teixido A, $M u “$ ller L.: Human exposure to dioxins through the diet in Catalonia, Spain: carcinogenic and non-carcinogenic risk. Chemosphere 2003;50:1193-1200.

32. US Environmental Protection Agency (USEPA). Supplemental Guidance for Developing soil Screening Levels for Superfund Sites. Office of Solid Waste and Emergency Response; Washington, DC, USA, OSWER 2001;9355: 4-24.

33. Howsam M, Jones KC.: Sources of PAHs in the environment. In: Neilson $A H$ (ed.) Handbook of environmental chemistry, PAHs and related compounds. Springer, Berlin, 1998; 3(1) :137-174.

34. Schoeny $R$, Poirier $K$.: Provisional guidance for quantitative risk assessment of polycyclic aromatic hydrocarbons, U.S. Environmental protection agency, office of research and development, Office of health and environmental assessment, Washington, DC,EPA/600/ R-93/089(NTISPB94116571),2016. https://cfpub.epa. gov/ncea/risk/recordisplay.cfm?deid $=49732$.

35. Lemieux $C L$, Long AS, Lambert IB, Lundstedt $S$, Tysklind $M$, White PA.: Cancer risk assessment of polycyclic aromatic hydrocarbon contaminated soils determined using bioassay-derived levels of benzo[a] pyrene equivalents. Environ Sci Technol. 2015;49:179805.

36. Wickramasinghe AP, Karunaratne DGGP, Sivakanesan $R$. : PM10-bound lung cancer risk of realistic different source scenarios, polycyclic aromatic hydrocarbons: Biological indicators, receptors and 'source-exposureeffect relationship. Chemosphere 2012;87:1381-7.

37. Jolly YN, Islam A , Akbar S.: Transfer of metals from soil to vegetables and possible health risk assessment. Springerplus 2013;2: 385.

38. Bureau of Water Quality Program Guidance WPDES, Guidance Number: 3400-2015-01, Wisconsin Department of Natural Resources, 2015. 
39. Ciemniak A, Witczak A Mocek K.: Assessment of trace element levels in Rhododendron honeys of Black Sea Region. Turkey J. Hazardous Materials 2013;156: 612618.

40. Lister LJ, Svendsen C, Wright J,. Hooper HL, Spurgeon DJ.: Modelling the joint effects of a metal and a pesticide on reproduction and toxicokinetics in Lumbricid earthworms. Environ Int. 2011;37:663-670.

41. Calderon J, Ortiz-Perez D, Yanez L, Díaz-Barriga F.: Human exposure to metals. Pathways of exposure, biomarkers of effect, and host factors. Ecotoxicol Environ Saf. 2003 Sept;56(1): 93-103.
42. Alloway B J.: Sources of heavy metals and metalloids in soils. Heavy metals in soils. Springer 2013; 11:50. DOI: 10.1007/978-94-007-4470-7 2.

43. Burdick AD, Davis JW, Liu KJ, Hudson LG, Shi H, Monske ML, Burchiel $S W$ : Benzo(a)pyrene quinones increase cell proliferation, generate reactive oxygen species, and transactivate the epidermal growth factor receptor in breast epithelial cells. Cancer Research.;2003;632(2):7825-7833.

Received: 08.01.2020

Accepted: 02.03.2020 